\title{
Correspondence
}

\section{Novel clinical pathways applied to cardiac surgery to improve outcome and to decrease perioperative resource utilization}

To the Editor:

We read with great interest the article by Novick et al. ${ }^{1}$ reporting on the impact of a specialized surgery recovery unit on outcome after cardiac surgery. Novick et al. confirm previous work which demonstrates that the creation of a specialized multidisciplinary recovery unit with $24 \mathrm{hr}$ on-site consultant coverage improves outcome. However, it may be open to some interpretation as to how differences between the improved composite outcome morbidity observed in isolated coronary artery bypass graft procedures might explain the unchanged composite outcome in isolated valve surgery, as the report does not present the changes with respect to each complication. This makes it somewhat uncertain as to which factor(s) were predominantly associated with each change in outcome. The change in cardiac surgical staffing during the second year of the program could have had an influence on complication rates, which may have introduced a bias in the observed composite outcomes between years 1 and 2. A further limitation of the study relates to the lack of standardization of the intraoperative anesthetic technique and surgical management.

It is also of interest to note that despite established ${ }^{2,3}$ and increasingly simple ${ }^{4}$ weaning protocols, time to tracheal extubation did not meet the criteria of fast-track cardiac anesthesia (i.e., tracheal extubation within eight hours postoperatively).

The benefits of re-organizing human resources, but also, introducing clinical pathways covering all aspects of anesthesia, surgery, nursing, and rehabilitation, are being increasingly recognized. Such pathways have been advocated for different types of surgery ${ }^{5}$ and have been shown to further improve postoperative outcomes. Clinical pathways might include protocols for nutritional support, mobilization, respiratory physiotherapy, removal of thoracic drains, etc. Changes introduced through such pathways might have an equally important impact on postoperative outcome, and may be less costly to implement, than the infra- structural changes presented in Novick's et al.'s study.

Changes continue to evolve in the perioperative management of patients undergoing cardiac surgery, to further improve outcomes, and to decrease resource utilization. We appreciate the work of Novick et al., in an effort to verify the effectiveness of a program with multimodal interventions. Interdisciplinary communication and exchange is equally important as the development of modern clinical pathways, which cover more than just respiratory care and weaning. The close interaction of all partners in the perioperative management of cardiac surgery patients, including surgeons, anesthesiologists, intensivists, nurses, respiratory physiotherapists, and nutritionists, is at the core and success of the implementation of these changes.

Thomas M. Hemmerling MD DEAA

Franco Carli MD

McGill University, Montreal, Canada

E-mail: thomashemmerling@hotmail.com

Accepted for publication April 24, 2008.

\section{References}

1 Novick RJ, Fox SA, Stitt LW, et al. Impact of the opening of a specialized cardiac surgery recovery unit on postoperative outcomes in an academic health sciences centre. Can J Anesth 2007; 54: 737-43.

2 Myles PS, Daly DJ, Djaiani G, Lee A, Cheng DC. A systematic review of the safety and effectiveness of fasttrack cardiac anesthesia. Anesthesiology 2003; 99: 982-7.

3 Cheng DC, Wall C, Djaiani G, et al. Randomized assessment of resource use in fast-track cardiac surgery 1-year after hospital discharge. Anesthesiology 2003; 98: 651-7.

4 Belii A, Pottecher J, Huntzinger J, Beydon L. Implementation of a weaning algorithm in postoperative cardiac ICU: simple enough to be implemented in a ventilator software. Ann Fr Anesth Reanim 2007; 26: 305.el-6.

5 White PF, Keblet H, Neal JM, Schricker T, Carr DB, Carli F; Fast-Track Surgery Study Group. The role of the anesthesiologist in fast-track surgery: from multimodal analgesia to perioperative medical care. Anesth Analg 2007; 104: 1380-96. 
Reply:

We thank Drs. Hemmerling and Carli for their letter and for their overall positive view of our paper. We embarked on this study because of the "natural experiment" provided by the provincially-mandated consolidation of two cardiac surgery units in London into a single heart centre in April 2005. We were pleased to note that the composite morbidity rate for our entire cohort decreased from $16.3 \%$ in the year prior to the merger, to $13.0 \%$ in the first year after the merger, a difference that was clinically and statistically significant. As Drs. Hemmerling and Carli have noted, most of the improved composite morbidity outcome was observed in patients undergoing isolated coronary artery bypass graft $(C A B G)$ surgery. They correctly pointed out that the composite morbidity rate after isolated valve surgery did not change between year 1 and year 2, even though the isolated valve surgery mortality rate decreased from $4.3 \%$ to $3.6 \%$. It should be noted, however, that the composite morbidity rate in combined CABG-palve patients decreased from $37.1 \%$ to $26.4 \%$ from year 1 to year 2, a value that bordered on statistical significance and had clinical importance.

Drs. Hemmerling and Carli indicated that it was "somewhat uncertain as to which factor(s) were predominantly associated with each change in outcome". Given the fact that this study reported on a "natural experiment", as opposed to a randomized controlled trial, it was very difficult to establish the precise causality among observed outcomes. Nonetheless, on multivariable modeling the pre-merger year was shown to be an independent predictor of death or major complications, with an odds ratio and $P$ value that were similar to other major risk factors such as a creatinine $>120 \mathrm{umol} \cdot \mathrm{L}^{-1}$ and diabetes. The possibility of bias is inherent in any prospective cohort study, especially in those with a before/after study design. This issue was reviewed extensively in the Discussion of our paper, which also highlighted the specific factors that we believe could have led to the improved outcomes that we demonstrated in the second year of the study.

Drs. Hemmerling and Carli's letter also mentioned the "lack of standardization of the intraoperative anesthetic technique and surgical management" and the fact that our median time to tracheal extubation was still $8.0 \mathrm{hr}$ in the second year of the study. We hereby confirm that our intraoperative anesthetic techniques and surgical procedures were as well standardized as those at any other academic health sciences centre, and that our experience reflects the "real world" conduct of contemporary cardiac anesthesiology and surgery practice. A recent systematic review of fast-track cardiac anesthesia encompassed in its operational definition the intention to promote early (< $10 \mathrm{hr}$ ) tracheal extubation. ${ }^{1}$ We are pleased to report that our median postoperative ventilation hours have continued to decrease successively from $8.0 \mathrm{hr}$, in the second year of our study to under $7.0 \mathrm{hr}$ presently. As Drs. Hemmerling and Carli correctly point out, comprehensive clinical pathways covering not only ventilator weaning, but also a host of postoperative care elements, are important as cardiac surgery units strive to improve outcomes and decrease resource utilization.

Richard J. Novick MD MSC FRCSC

Stephanie A. Fox BSC RRCP

Larry W. Stitt MSc

Ron Butler MD MSC FRCPC

Mary Kroh BsCN

Christina Hurlock-Chorostecki MSCN ACNP CNCC(C)

Chris Harris RRT

Davy C.H. Cheng MD MSc FRCPC

London Health Sciences Center, University Hospital, London, Canada

E-mail: richard.novick@lhsc.on.ca

\section{Reference}

1 Myles PS, Daly DJ, Djaiani G, Lee A, Cheng DC. A systematic review of the safety and effectiveness of fast-track cardiac anesthesia. Anesthesiology 2003; 99: 982-7.

\section{Skin infection and necrosis after subcutaneous infiltration of propofol in the intensive care unit}

To the Editor:

Microorganisms grow rapidly in propofol, and extrinsic contamination of propofol is thought to be a source of postoperative sepsis and wound infection. ${ }^{1}$ We describe a case in which propofol is believed to have caused skin necrosis.

A 70-yr-old male underwent coronary artery bypass grafting and repair of the aortic and mitral valves. His postoperative course was complicated by acute renal failure, encephalopathy, Pseudomonas aeruginosa pneumonia, methicillin resistant staphylococcus epidermidis (MRSE) bacteremia, and respiratory failure that required prolonged mechanical ventilation. $\mathrm{He}$ was sedated with a continuous intravenous infusion of propofol via a $14-\mathrm{G}$ catheter inserted on the dorsum of his right hand. The propofol infusion was handled according to the manufacturer's directions. Three days 